\title{
Increased frequency of lymphocytic mitotic non-disjunction in recurrent spontaneous aborters
}

\author{
RICHARD C JUBERG*†, JUDY KNOPS*, AND PHILIP N MOWREY* \\ From the *Department of Medical Genetics and Birth Defects, Children's Medical Center, and the \\ †Department of Pediatrics, Wright State University, School of Medicine, Dayton, Ohio, USA.
}

SUMMARY Hypermodal chromosomal spreads occurred significantly more frequently in lymphocytes from couples with recurrent spontaneous abortion than from comparison populations. Previously, we reported a similarly increased frequency in couples with aneuploid offspring. Considering the frequency of aneuploidy among first trimester spontaneous abortions, we suggest that there may be a sub-population of persons predisposed to non-disjunction among couples with reproductive wastage.

Sporadic, non-modal chromosomal spreads may be either hypomodal, in which the number of chromosomes is less than the modal number, or hypermodal, with more than the mode. Hypermodals occur less frequently than hypomodals in routine lymphocytic preparations. The hypomodal spread has generally been considered to be a technical artefact resulting from random loss during the preparation of slides by the splash technique. In contrast, the less frequently occurring hypermodal spread may originate differently and be meaningful. Previously, we called attention to the possibility of their non-random occurrence among the subjects studied in a clinical cytogenetic laboratory. ${ }^{12}$

We showed that the frequency of sporadic, hypermodal spreads from the parents of aneuploid offspring significantly exceeded that found in comparison populations. We suggested mitotic nondisjunction as the origin of these spreads after eliminating other possibilities. Predisposition to lymphocytic mitotic non-disjunction in vitro might reflect an inherent tendency towards nondisjunction, including meiotic non-disjunction which leads to an aneuploid conceptus. An aneuploid conceptus will be either liveborn, with considerable probability of physical and mental abnormalities, or spontaneously aborted. The outcome is particularly dependent on the type of aneuploidy.

Here we show that the frequency of sporadic, hypermodal spreads observed in recurrent spontaneous aborters significantly exceeded the frequency in suitable comparison groups. The fre-

Received for publication 3 April 1984. Accepted for publication 8 May 1984. quency was, not surprisingly, comparable to that among couples with aneuploid offspring.

\section{Materials and methods}

Chromosomal spreads were obtained from the cultured lymphocytes of 717 subjects during 65 months in a diagnostic cytogenetic laboratory. The patients included (1) those clinically suspected of having a chromosomal abnormality; (2) those phenotypically abnormal because of malformation, mental retardation, learning disability, or some combination of these; and (3) those phenotypically normal who were studied for prognostic reasons because of their relationship to a chromosomally abnormal subject or because of their reproductive record.

We established lymphocytic cultures either from whole blood or leucocyte rich plasma, and we harvested the cells by standard techniques or cell synchronisation or both. ${ }^{3}$ We analysed chromosomal spreads according to $G$ band quality ${ }^{4}$ and chromosomal dispersion. Generally, to evaluate the possibility of mosaicism, we studied 20 to 32 spreads. $^{5}$

At the time of discovery of a non-modal spread, there was no such study taking place in our laboratory. Therefore, there was no reason for the microscopists to make any association with the type of subject undergoing clinical analysis. The designation of a hypermodal spread required concordance by at least two experienced laboratory workers. The microscopists generally had little knowledge of the reason for the study of any patient at any time and, thus, could not have had any bias in the selection of 
spreads. Consequently, there could not possibly have been a prejudice to locate hypermodals or hypomodals in any particular type of subject. Our inquiry about the frequency of non-modal spreads and about the type of subject in whom they occurred used observations made before the review of records which comprised this research.

For retrospective analysis of the relationship between subjects and type of hypermodal spreads, we separated three groups from the total of 717 subjects: (1) 42 parents of aneuploid offspring; (2) 31 subjects with a history of recurrent spontaneous abortion; and (3) 72 adults, shown to be $46, \mathrm{XX}$ or $46, X Y$, who had been studied cytogenetically in the course of genetic counselling or investigation of infertility, or for having a malformed child who was not known to have either a chromosomal abnormality or a diagnosis. The aneuploid offspring of the first group were: 13 with +21 , two with +18 , two with +13 , two with $X X Y$, one with $X Y Y,+13$, one with $\mathrm{XXX}$, and one with $\mathrm{XXXXY}$. The mean number of spontaneous abortions among the second group was $2 \cdot 87$ for 16 females.

The comparison group of 72 adults included only subjects physically and mentally capable of parenthood and excluded (1) anyone who had produced an offspring known, or strongly suspected, to have chromosomal abnormality, and (2) any person who had a reproductive record that included fetal wastage. This comparison group of 72 adults excluded all subjects with a chromosomal structural abnormality (for example, a balanced translocation) but included those with chromosomal variants, as did each of the other groups. The mean age of the 72 adults comprising the comparison group was quite similar to that of each of the two study groups, happened to be between them, and differed from neither significantly.

\section{Results}

Approximately $6 \%$ of the spreads of all subjects were hypomodal. We examined the general chromosomal distribution in a hypomodal spread and identified the loss. The deficiencies involved all chromosomes at frequencies nearly inversely proportional to relative chromosomal length. We attributed the deficiencies in the sporadic hypomodals to loss during slide preparation.

At least two microscopists examined every hypermodal spread to evaluate the distribution, to identify the type of additional chromosome, and to compare it, or them in the few instances of two additional chromosomes, to all others in the spread. We also compared homologues for their similarity of staining, position within the spread, and contracted state. Such microscopic and photomicroscopic comparisons provided the bases for discounting the spurious hypermodal spread where the contraction of one chromosome was strikingly different from its homologues, or where the peripheral position of a chromosome argued against its belonging in that spread.

Table 1 shows that there were 44 hypermodals among 717 subjects and their 21841 spreads; therefore, the frequency was 1 in 496 . There was no evident temporal, sequential, or personnel pattern to the discovery of these spreads. Cultures from 34 of the 717 subjects had one hypermodal spread, five had two, and none had more than two. These were observed in 22 males and 17 females.

Table 2 shows the distribution of the hypermodal spreads by the number of additional chromosomes $(1,2$, or 3$)$, by the chromosomal number, and by the sex. Hypermodality occurred among most of the autosomes in both sexes; however, $\mathrm{X}, \mathrm{Y}$, and 21 were most frequently involved. The five subjects with two hypermodals each were (1) a male with two spreads with an extra Y; (2) two females with two spreads with an additional X; (3) a female with one +21 and one $+\mathrm{i}(11 \mathrm{q})$; and (4) a male with one spread with two additional chromosomes 19 and another with an unidentified fragment.

Hypermodal spreads occurred at a frequency of

TABLE 1 Comparison of frequencies of hypermodal chromosomal spreads in different groups.

\begin{tabular}{|c|c|c|c|c|c|c|}
\hline Subject group & No & $\begin{array}{l}\bar{x} \text { Age } \\
(y)\end{array}$ & $\begin{array}{l}\text { Total } \\
\text { spreads }\end{array}$ & $\begin{array}{l}\text { Total } \\
\text { hypermodals } \neq\end{array}$ & $\begin{array}{l}\text { Subjects } \\
\text { with } \\
\text { hypermodals }\end{array}$ & Frequency \\
\hline $\begin{array}{l}\text { A All subjects } \\
\text { B Parents of aneuploids* } \\
\text { C Parents with abortions* } \\
\text { D All except B and C } \\
\text { E Comparison parents* } \dagger\end{array}$ & $\begin{array}{r}717 \\
42 \\
31 \\
644 \\
72\end{array}$ & $\begin{array}{l}12 \cdot 2 \\
29 \cdot 4 \\
30 \cdot 1 \\
10 \cdot 3 \\
29 \cdot 6\end{array}$ & $\begin{array}{c}21841 \\
1391 \\
925 \\
19525 \\
1897\end{array}$ & $\begin{array}{r}44 \\
11 \\
8 \\
25 \\
1\end{array}$ & $\begin{array}{r}39 \\
9 \\
7 \\
23 \\
1\end{array}$ & $\left.\begin{array}{l}1 / 496 \\
1 / 127 \S \\
1 / 116 \\
1 / 781 \\
1 / 1897\end{array}\right]$ \\
\hline
\end{tabular}

- All parents were $46 . \mathrm{XX}$ or $\mathrm{XY}$

† Adults lacking aneuploid progeny or multiple abortions.

$\ddagger$ Addition of $\mathrm{X}$ or $\mathrm{Y}$ occurred in 0.64 of the hypermodals in $\mathrm{B}, 0.37$ in $\mathrm{C}, 0.17$ in $\mathrm{D}$, and 0 in $\mathrm{E}$

$\S \mathrm{p}<0.01, \chi^{2}$ with $1 \mathrm{df}$.

\| Not significant. 
TABLE 2 Distribution of hypermodal spreads by number of additional chromosomes, by chromosome number, and by sex.

\begin{tabular}{|c|c|c|}
\hline \multirow[t]{2}{*}{ Chromosome No } & \multicolumn{2}{|c|}{ No of hypermodal spreads } \\
\hline & Males & Females \\
\hline \multicolumn{3}{|c|}{ I additional chromosome } \\
\hline+3 & $\cdots$ & 1 \\
\hline+4 & 1 & $\cdots$ \\
\hline+5 & $i$ & $\cdots$ \\
\hline+6 & 1 & $\cdots$ \\
\hline+7 & 2 & $\cdots$ \\
\hline+8 & 3 & $\cdots$ \\
\hline+11 & 1 & $2^{*}$ \\
\hline+12 & 2 & $\cdots$ \\
\hline$+X$ & $\cdots$ & 7 \\
\hline+13 & 1 & $\cdots$ \\
\hline+14 & $\cdots$ & $1+$ \\
\hline+16 & 1 & $\cdots$ \\
\hline+17 & $\cdots$ & 1 \\
\hline+18 & 1 & $\cdots$ \\
\hline+19 & $\ldots$ & 1 \\
\hline+20 & $\ldots$ & $\cdots$ \\
\hline+21 & $\cdots$ & 3 \\
\hline+22 & $\cdots$ & 1 \\
\hline$+\mathrm{Y}$ & 5 & $\cdots$ \\
\hline +fragment $\ddagger$ & 3 & $\cdots$ \\
\hline \multicolumn{3}{|c|}{2 additional chromosomes } \\
\hline$+(3,6)$ & $\cdots$ & 1 \\
\hline$+(12,13)$ & $\cdots$ & 1 \\
\hline$+(X, X)$ & $\cdots$ & 1 \\
\hline$+(19,19)$ & 1 & $\cdots$ \\
\hline \multicolumn{3}{|c|}{3 additional chromosomes } \\
\hline$+(\mathrm{Y}, \mathrm{Y}, \mathrm{Y})$ & 1 & $\cdots$ \\
\hline Total & 24 & 20 \\
\hline
\end{tabular}

* Both +i(11q).

† A D group not further identified.

$\ddagger$ Not further identified.

1 in 127 among the parents of aneuploid offspring. By comparison, hypermodals appeared at a frequency of 1 in 781 among all subjects after removal of the parents of aneuploids and persons with recurrent spontaneous abortion (table 1 , line $D)$. The difference was highly significant $\left(p<0 \cdot 001, \chi^{2}\right.$ with $1 \mathrm{df}$ ). Table 1 also shows that the frequency of hypermodal spreads among the comparison parents, 1 in 1897 , was only about $1 / 15$ that of the parents of the aneuploid offspring. This difference was highly significant $\left(p<0.001, \chi^{2}\right.$ with $\left.1 \mathrm{df}\right)$.

Hypermodal spreads occurred with a frequency of 1 in 116 among the persons with recurrent spontaneous abortion, which was significantly different both from the remainder of subjects $\left(p<0.001, \chi^{2}\right.$ with $1 \mathrm{df}$ ) and from the group of comparison parents $\left(\mathrm{p}<0.001, \chi^{2}\right.$ with $\left.1 \mathrm{df}\right)$.

\section{Discussion}

The cause of meiotic non-disjunction, which results in aneuploid conceptions, remains unknown despite $\stackrel{?}{-}$ extensive investigation during the past 20 years. $\overrightarrow{\vec{c}}$ Possible causes have been considered: (1) recessive genes; (2) the infectious hepatitis virus; (3) the $\frac{}{0}$ autoimmune effect of Hashimoto thyroiditis; (4) $\bar{\sigma}$ ionising radiation; (5) $\alpha_{1}$-antitrypsin variants; (6) $\vec{\nabla}$ medications and chemicals; (7) the $\mathrm{pH}$ at the site of $\frac{\mathrm{Q}}{2}$ fertilisation; (8) chromosomal variants; and (9) क aging of two forms, that is, in years of life and in $\overrightarrow{0}$ hours of the ovum before fertilisation. ${ }^{6}$ The problem $\overrightarrow{\vec{H}}$ of autosomal or sex chromosomal aneuploidy $\vec{\omega}$ weighs heavily on families and on society considering that it occurs with a frequency of about 5.6 in 0 1000 live births ${ }^{7}$ and over 50 in 100 first and second i N trimester spontaneous abortions. ${ }^{8}$

Advancing parental age is recognised as increasing the risk of producing an aneuploid livebirth. Women older than 35 years have more than a $0.5 \%$. chance of producing a child with a clinically signifi- $\rightarrow \pi$ cant chromosomal abnormality. ${ }^{9}$ However, fewer $\stackrel{\mathbb{O}}{\circ}$ than $5 \%$ of births occur to women over 35 years ${ }^{10} \frac{O}{2}$ and, similarly, fewer than $50 \%$ of trisomy 21 births come from such women. ${ }^{11}$ Moreover, apart from the $\vec{\theta}$ uncommon carrier of relevant types of transloca- $\infty$ tions and possibly certain chromosomal variants, no. female or male under 35 years can now be recognised as being at increased risk for primary meiotic non-disjunction.

In addition to the parents of a liveborn aneuploid $\stackrel{\varrho}{\circ}$ child, couples experiencing spontaneous abortion $\stackrel{\mathbb{Q}}{\varrho}$ constitute another group with meiotic non- $\overrightarrow{\overrightarrow{0}}$ disjunction. In an earlier report based on fewer $\frac{3}{3}$ studies, ${ }^{2}$ we showed that the frequency of hyper- $\vec{\partial}$ modal spreads among couples having recurrent spontaneous abortion exceeded that of comparison groups, and the present data enlarge and confirm that finding. Allen et al ${ }^{12}$ have observed a similarly $\dot{0}$ increased frequency of sporadic hypermodals among persons referred for spontaneous abortions. How- 8 ever, in their report a small group of parents of 3 aneuploids did not show hypermodals.

We believe that couples who have recurrent spontaneous abortion and whose lymphocytic cultures reveal a sporadic hypermodal may constitute a

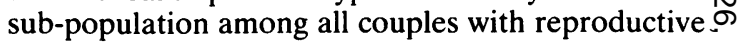
wastage who may be at increased risk of meiotic $\mathbb{N}$ non-disjunction. Perhaps they have an inherent $\underset{\omega}{N}$ tendency to non-disjunction. Consequently, we believe that they should be considered for antenatal investigation and appropriately counselled, regard- $\frac{0}{\Phi}$ less of age.

We thank Dr Richard Stallard for helpful comments, Patricia Beltz, Nancy R Haney, and Patti A $\stackrel{\mathbb{D}}{\Omega}$ Frank for technical assistance, and Merilace Huff $\stackrel{\mathrm{\Phi}}{\mathrm{D}}$ and Patty Styron for processing data. 


\section{References}

${ }^{1}$ Stallard R, Haney NR, Frank PA, Styron P, Juberg RC. Leukocyte chromosomes from parents of cytogenetically abnormal offspring: preliminary observations. Cytogenet Cell Genet 1981:30:50-3

2 Stallard R, Haney NR, Frank PA, Mowrey P, Knops J, Juberg RC. Detecting inherent parental tendency to nondisjunction. Am J Hum Genet 1981;33:123A.

${ }^{3}$ Yunis JJ. High resolution of human chromosomes. Science 1976;191:1268-70.

${ }^{4}$ Seabright M. A rapid banding technique for human chromosomes. Lancet 1971;ii:971-2.

5 Turpin R, Lejeune J. Techniques d'etude des chromosomes humains. In: Les chromosomes humains. Paris: GauthierVillars, 1977:43-4.

${ }^{6}$ Juberg RC, Goshen CR, Sholte FG. Socioeconomic and reproductive characteristics of the parents of patients with the $\mathrm{G}_{1}$-trisomy syndrome. Soc Biol 1973;20:404-15.

${ }^{7}$ Hook EB, Hamerton JL. The frequency of chromosome abnormalities detected in consecutive newborn studiesdifference between studies-results by sex and by severity of phenotypic involvement. In: Hook EB, Porter IH, eds. Population cytogenetics. New York: Academic Press, 1977:63-79.
${ }^{8}$ Kajii T, Ferrier A, Niikawa N, Takahara H, Omaha K, Avirachan S. Anatomic and chromosomal anomalies in 639 spontaneous abortuses. Hum Genet 1980;55:87-98.

${ }^{9}$ Hook EB, Cross PK, Schreinemachers DM. Contemporary estimates of maternal age specific rates of Down syndrome and other trisomies in livebirths (in absence of selective abortion) using regression smoothed rates from prenatal diagnostic studies adjusted for spontaneous fetal death after amniocentesis. Am J Hum Genet 1982;34:128A.

${ }^{10}$ Sepe SJ, Marks JS, Oakley GP, Manley AF. Genetic services in the United States, 1979-1980. JAMA 1982;248:1733-5.

11 Evans JA, Hunter AGW, Hamerton JL. Down syndrome and recent demographic trends in Manitoba. $J$ Med Genet 1978;15:43-7.

12 Allen E, Rolland D, Ching E, Morse B. Frequency of sporadic chromosomally abnormal cells in patients referred for multiple spontaneous abortions. Am J Hum Genet 1982;34:116A.

Correspondence and requests for reprints to $\mathrm{Dr}$ Richard C Juberg, Frederick A White Center for Ambulatory Care, Wright State University, Dayton, Ohio 45435, USA. 